\title{
Solving Contaminant and Unexpected Material Problems in Drugs and Medical Devices Using Microscopy Methods - An Overview
}

\author{
Debra Joslin ${ }^{1}$
}

1. McCrone Associates, Westmont, IL USA

Foreign material and contaminants are of concern in most consumer products, but are especially so in the pharmaceutical industry due to regulatory requirements. Many of these foreign materials are particulates, and are most easily identified using microscopy methods, including light microscopy (LM), scanning electron microscopy with energy dispersive X-ray spectrometry (SEM/EDS), and transmission electron microscopy (TEM), in combination with micro Fourier transform spectroscopy (IR) and other analytical methods. Identification of these materials is critical to determining the source(s) of contamination and documenting their origin for regulatory compliance purposes.

Foreign material can arise from myriad sources including (but not limited to) machine parts, glassware, stoppers, filters, biological entities, clothing, paper products, and chemical reactions. The possibilities for origin are endless. In the case of particulate, bulk analysis methods are inappropriate due to the miniscule concentration of the contaminant in the overall sample. As an example, fibers can often be identified by polarized light microscopy (PLM). Metals and other inorganic materials are generally analyzed by SEM/EDS, while solid organic materials are mostly analyzed using IR unless there are inorganic fillers or other environmental contamination in the material.

How is particulate identification accomplished? In order to minimize the possibility of cross contamination during isolation and preparation for analysis, the particles of interest are handled within a clean room environment. Typically, foreign material in solid samples is first observed using a stereo microscope, isolated from the sample using custom tools, and then characterized optically using a stereo microscope and possibly a polarized light microscope. Example properties that might be observed include mechanical response to probing, color, texture, reflectivity, presence/absence of birefringence, and refractive index.

Foreign material in liquid samples can be more complex. If the particulate is large enough to remove from the liquid directly, it is removed and characterized by LM. If not, the particulate must be filtered from the liquid. The particulate is characterized on the filter, and if possible, removed from the filter and treated as if it were an independent particle. If the particulate is too thin to be isolated from the filter, it sometimes can be analyzed on the filter. This may lead to ambiguous results; in that case, proprietary methods can be used to isolate the material for analysis by SEM/EDS or TEM.

With medical devices, two general scenarios arise: the issue of contamination during production, and failure during in vivo use. In the second case, additional engineering control measures for protection of employees are required (e.g. the use of a bio-safety cabinet or facility unless the samples are sterilized and delivered with a certificate of sterilization. Isolation of the foreign material and preparation for analysis is otherwise the same.

Figures 1 through 3 illustrate several of these points. 


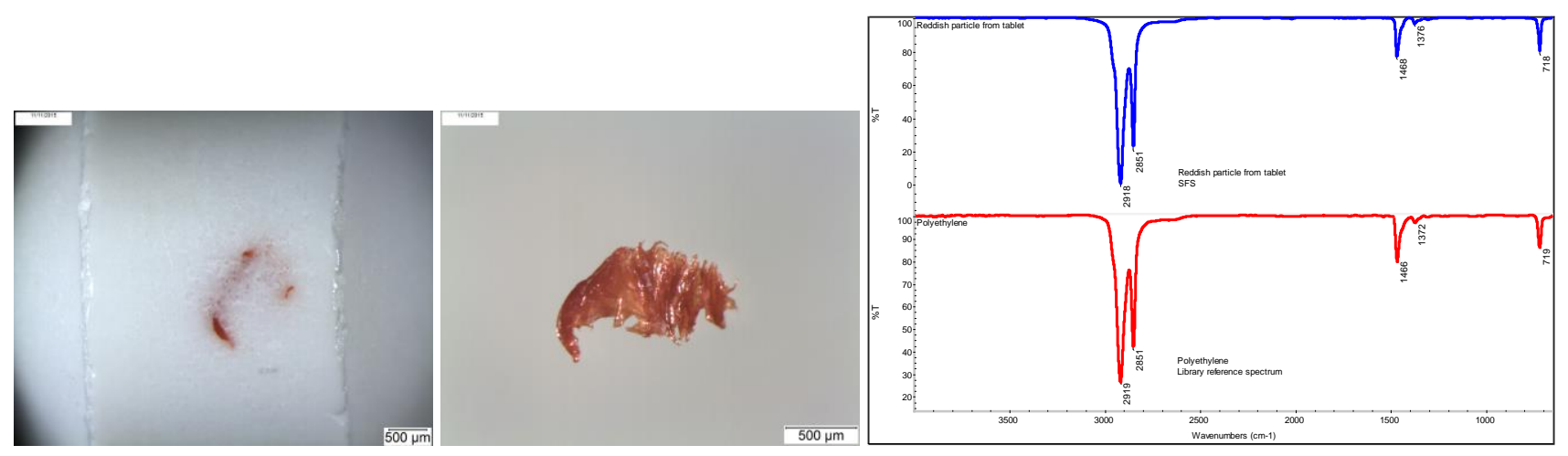

Figure 1. The reddish particle in the tablet was isolated, characterized using LM, and identified as polyethylene using IR.
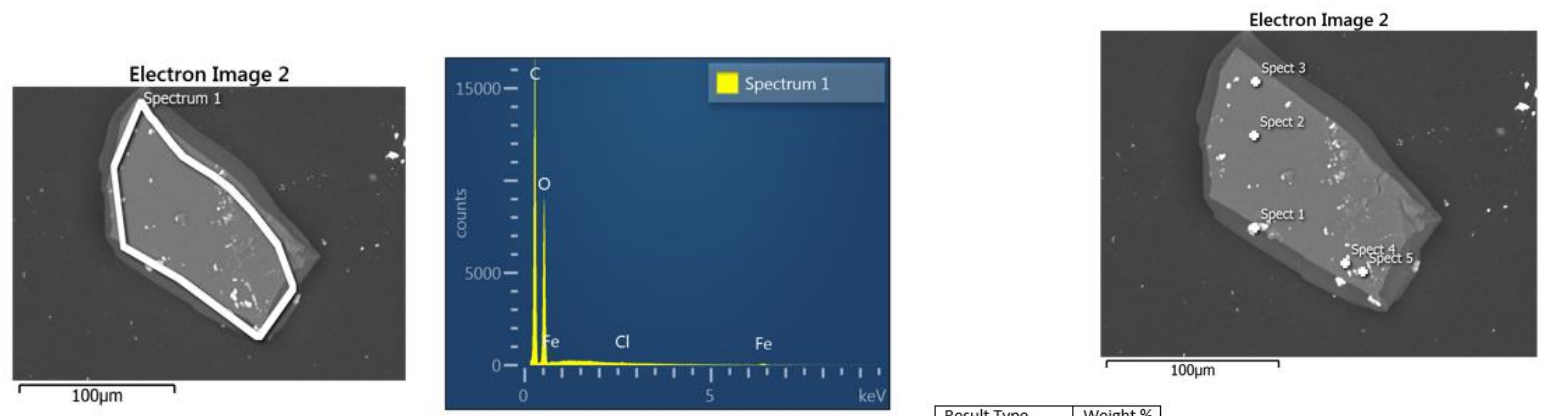

\begin{tabular}{|l|c|r|r|r|}
\hline \multicolumn{1}{|c|}{ Element } & Line Type & \multicolumn{1}{c|}{ Wt\% } & \multicolumn{1}{c|}{ Wt\% Sigma } & \multicolumn{1}{c|}{ Atomic \% } \\
\hline $\mathrm{C}$ & K series & 54.97 & 0.22 & 62.34 \\
\hline $\mathrm{O}$ & K series & 43.87 & 0.22 & 37.35 \\
\hline $\mathrm{Cl}$ & K series & 0.12 & 0.03 & 0.05 \\
\hline $\mathrm{Fe}$ & K series & 1.04 & 0.09 & 0.25 \\
\hline Total: & & 100.00 & & 100.00 \\
\hline
\end{tabular}

\begin{tabular}{|l|l|}
\hline Result Type & Weight \% \\
\hline
\end{tabular}

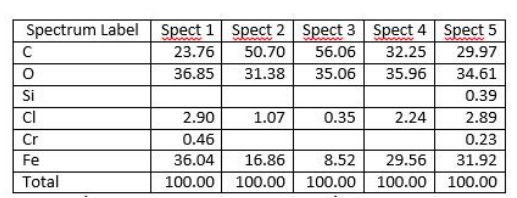

Figure 2. SEM/EDS analysis of a brown contaminant particle with inclusions revealed in the backscattered electron image. The bulk of the particle is organic; the inclusions are iron-rich. The presence of chlorine indicates possible corrosion.
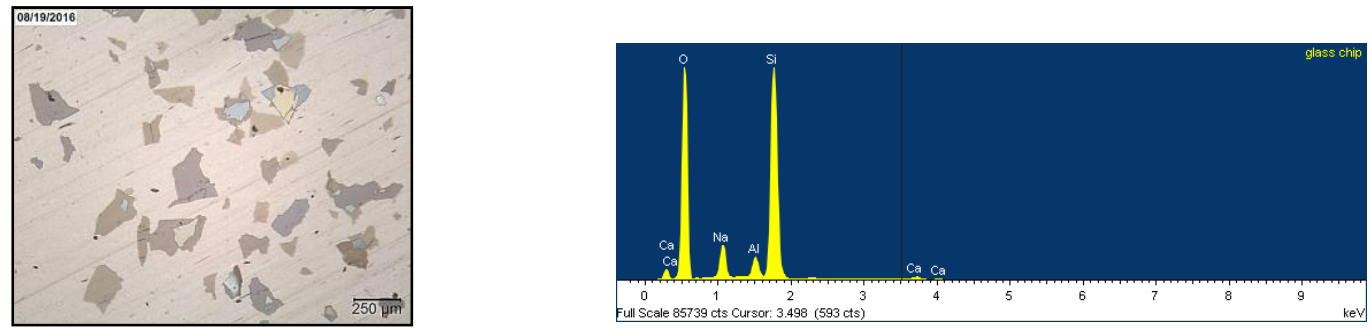

Figure 3. Glass delamination flakes on a polycarbonate filter, oblique lighting. The EDS spectrum is consistent with glass. 